\title{
Wide-field mid-infrared hyperspectral imaging by snapshot phase contrast measurement of optothermal excitation
}

Tao Yuan, ${ }^{1,2, \dagger}$ Miguel A. Pleitez, $, 1,2, \dagger, *$ Francesca Gasparin,, ${ }^{1,2}$ Vasilis Ntziachristos ${ }^{1,2, *}$

${ }^{1}$ Technical University of Munich, School of Medicine, Center for Translational Cancer Research (TranslaTUM), Chair of Biological Imaging, D-81675, Munich, Germany

${ }^{2}$ Helmholtz Zentrum München (GmbH), Institute of Biological and Medical Imaging, D-85764, Neuherberg, Germany

†These authors contributed equally to this work

${ }^{*}$ Corresponding author. Email: bioimaging.translatum@tum.de

Table of contents

Figure S1. Characterization of the condenser-free phase contrast microscope (CPCM).

Figure S2. Simulation of MIR irradiation FOV and its comparison with experimental result. 
To establish the relationship between phase shift and intensity, we modeled the wavefront propagation using the microscope with support of a SDK, PROPER ${ }^{1}$.

a

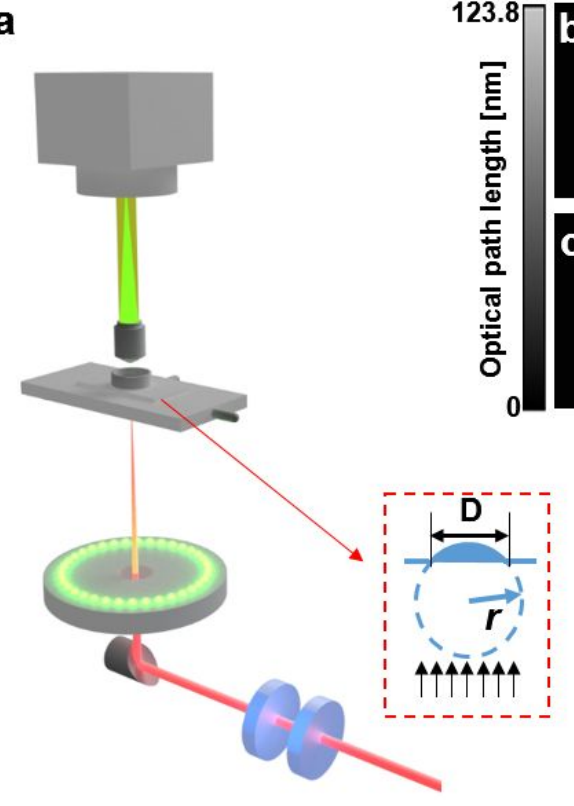

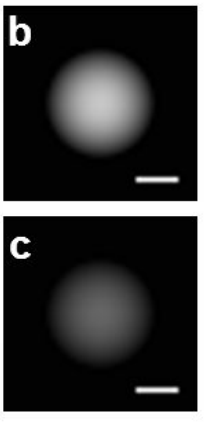
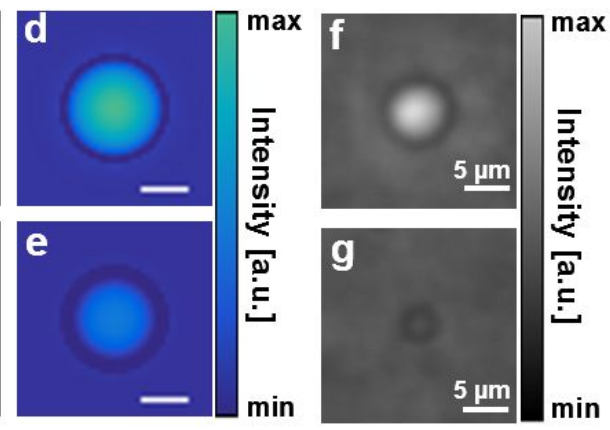

h

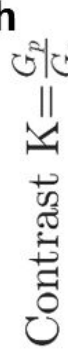

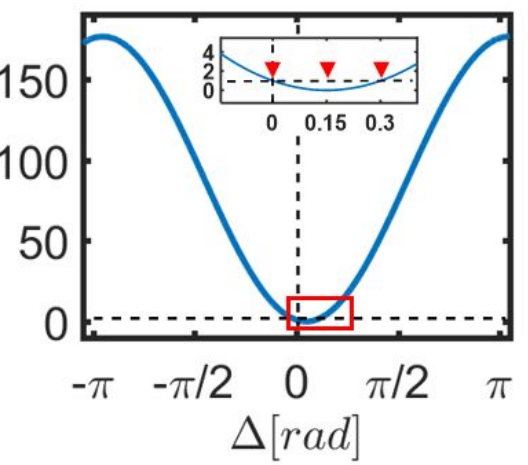

Figure S1. Characterization of the condenser-free phase contrast microscope (CPCM). a, System configuration of the CPCM, and a TAG drop model (side view, blue shaded area) with diameter $D$ $=30 \mu \mathrm{m}$. The drop model is simplified as a part of a virtual sphere with radius of $r$. When light from below travels through the droplet, the phase of light's wavefront retards according to the thickness of the droplet. The light passing through the drop travels a longer optical path length $(n-1) d$ compared to which passing through the surrounding area, where $n$ is the refractive index of TAG droplet, and $d$ is the droplet thickness. This optical path difference corresponds to phase retard of $\frac{2 \pi(n-1) d}{\lambda}$, where $\lambda$ is the wavelength of light (phase illumination). b,c, The top view of two drops with different thickness $(r=0.5$ and $r=1 \mathrm{~mm}$; D $=30 \mu \mathrm{m}$ for both, scale bar: $10 \mu \mathrm{m})$. d,e, Corresponding images acquired by simulation of CPCM. f,g, Experimental result (MIR-OFF phase image) of two TAG drops from our setup. Comparison of (d) and (e) indicates that longer optical path difference leads to stronger light intensity in the center. As similar to the experiment, the image of the drop is surrounded by a dark halo, which relates to short optical path length from the drop edge. $\mathbf{h}$, The quantitative relation between phase retard $\Delta$ and contrast $K=\frac{G_{p}}{G_{s}}$ based on a mathematical model provided by ref $^{2}$ as a cross-check method for our simulation. Here, $G_{p}$ is the intensity of the investigated point (sample or background), $G_{s}$ is the intensity of the surrounding area (background), positive direction of $\Delta$ axis represents the phase retard of the investigated point relative to the surrounding area. As seen in the phase retard ranging from 0 to $\pi \mathrm{rad}$, the contrast increases as the phase retards. According to this, it is possible to establish a quantitative relation between the intensity image and the retarded phase. In the insert plot, the negative contrast range (0-0.3 rad) corresponding to short optical path length is confirmed by our simulation (d-e), as well as experimental result (f-g), where the edge of the drops shows dark halo. The dark halo provides a 
way to predict the absolute phase retard from the intensity image. Specifically, the darkest points of the halo correspond to a phase retard of $0.15 \mathrm{rad}$, as indicated by the minimum point in (h).
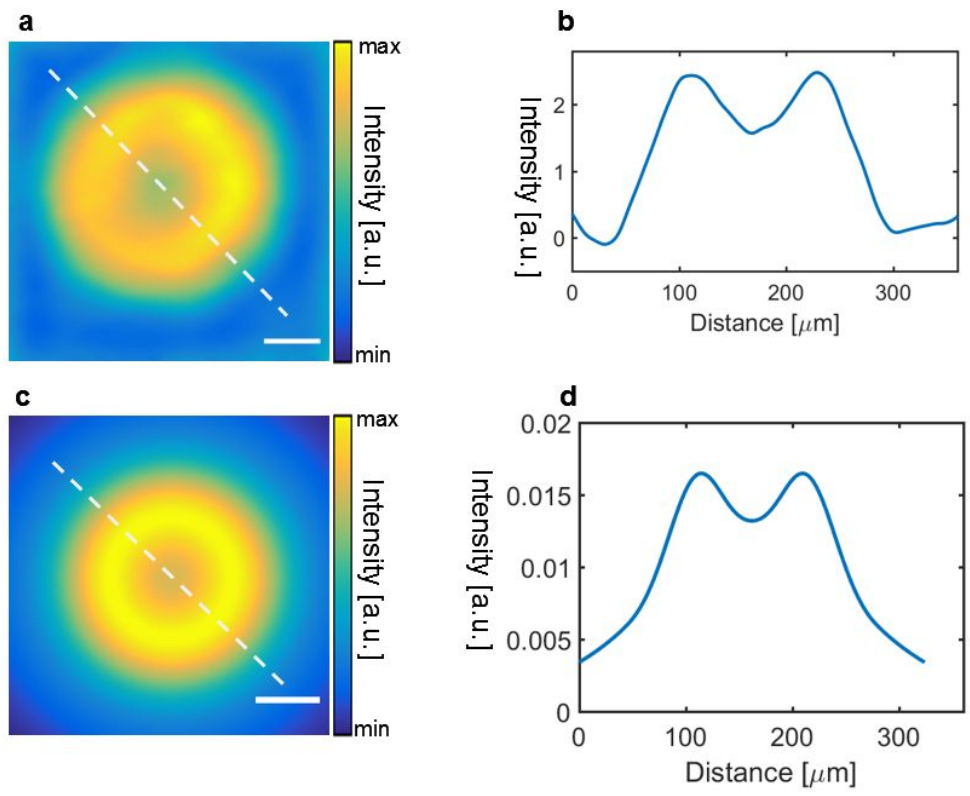

Figure S2. Simulation of MIR irradiation FOV and its comparison with the experimental result (scale bar: $100 \mu \mathrm{m}$ ). a, Experimental result (CPCM image) as MIR irradiating the water (smoothed image). b, Line profile across the MIR irradiation spot [dashed line on (a)]. c, Simulation result (CPCM image) by a Gaussian sample (the optical path length distribution is a Gaussian distribution, assuming that MIR optothermal heat is a Gaussian distribution). d, Line profile across the image [dashed line on (c)]. Both the experimental and simulation results show doughnut-shaped contrast provided by CPCM, suggesting that CPCM can provide a Gaussian peak suppressed FOV.

\section{References}

(1) Krist, J. E., PROPER: an optical propagation library for IDL, in Optical Modeling and Performance Predictions III. (International Society for Optics and Photonics, 2007), 66750P.

(2) Bennett, A. H.; Jupnik, H.; Osterberg, H.; Oscar, W., Phase microscopy: principles and applications, Wiley: 1951. 